\title{
Let Us Aggressively Confront the Coronavirus Pandemic
}

\author{
John Somberg
}

The coronavirus due to its infectivity, contagious from otherwise healthy appearing individuals and high mortality, poses a serious threat to world health and the world economy. The medical community needs to approach the pandemic with urgency and with approaches that would not ordinarily be taken with a less urgent threat.

\section{Therapeutics}

Thus far, the only approach to limit the virus is public health measures: social distancing, travel bans and quarantines. More is urgently needed to combat the virus. Some therapeutic modalities have been suggested including: hydroxychloroquine/ chloroquine, chloroquine plus azithromycin, as well as a number of anti-viral compounds, as well as monoclonal antibodies. These therapies need to be divided into those employing established drugs and those new therapeutic entities. Repurposed therapies should go right into an efficacy trial given their urgent need. There are a number of adaptive trial designs that should be employed. One simple one is "play the winner", a very easy trial to carry out that would provide information on efficacy. Trials are needed in patients with mild symptoms, those with more pronounced disease with moderate symptoms, but not needing intensive care and trials in intubated patients in the intensive care unit. Given the tens even hundreds of thousands of patients available, recruitment should be rather straightforward. One could randomize by patient, but to simplify the process randomization could be by hospital, or even by region. Using simple, creative approaches could expedite these types of trials.

New or unapproved therapies would need to have more safety information obtained, but even with unapproved drugs, treatment in severely ill patients could be expedited given the lack of available therapies. There is no need for compassionate therapies, and all patients could be entered into clinical trials in a time of national emergency, thus enabling rapid results being obtained. Those who show no response could be randomized to alternative therapies being studied.

Manuscript submitted April 7, 2020, accepted April 14, 2020

Cardiology \& Pharmacology, Rush University, Chicago, IL 60612, USA. Email: John_Somberg@rush.edu

doi: https://doi.org/10.14740/cr1065

\section{Vaccine Therapy}

The only approach that will effectively terminate the economic and health impact of coronavirus is to develop an effective vaccine. While the virus has may antigenic targets, vaccine development for lasting effectiveness may be an issue, given the virus's ability to change antigenic determinants. Still a vaccine that is effective against this version of the virus would be a major advance. Vaccines development has become less laborious, employing the known genetic makeup of the virus and existing vaccine platforms. The process has already led to at least two vaccines going into clinical trials. What is disheartening is to hear scientific advisors, regulators and companies talking of 12 - 18 months to develop a vaccine. In normal times, this is the requisite and acceptable development timeline. But in the midst of a most serious pandemic, a year and a half is too long. When a vaccine is found to elicit antibodies in quantities expected to be clinically useful, the vaccine should be made available to those who want to make use of it and especially high-risk patients who are at the greatest risk of death. While there may be unexpected and possibly serious toxicities, in this time of crisis, those risks should be allowed to be accepted by individuals, especially those at high risk.

\section{Meeting the Challenge}

With expedited clinical trials, early introduction of therapeutic entities that show initial efficacy and the rapid development of vaccines, we hopefully can combat the coronavirus effectively.

\section{Acknowledgments}

None to declare.

\section{Financial Disclosure}

None to declare.

\section{Conflict of Interest}

None to declare. 